\title{
Impact of genetic selection on feed efficiency on carcass traits in Irish beef cattle
}

\author{
J.J. Crowley ${ }^{1,3}$, R.D. Evans ${ }^{2}$, D.P. Berry ${ }^{1}$
}

${ }^{1}$ Teagasc, Moorepark Dairy Production Research Centre, Fermoy, Cork, Ireland, ${ }^{2}$ Irish Cattle Breeding Federation, Bandon, Cork, Ireland, ${ }^{3}$ School of Agriculture, Food Science and Veterinary Medicine, College of Life Sciences, University College Dublin, Belfield, Dublin 4, Ireland

Email:john.j.crowley@teagasc.ie

Introduction Feed is the largest variable cost on farms and there is much interest in selecting for improved feed efficiency (FE). However, prior to recommending selection on any trait, the expected response to selection on other economically important traits needs to be quantified. The objective of this study was to quantify the genetic relationship between feed efficiency and carcass fat (CF), carcass conformation (CC) and carcass weight (CW) in Irish beef cattle.

Materials and methods Data for this analysis originated from two separate sources. The first data set consisted of feed intake (FI) and bodyweight (BW) records collected at the Irish bull performance test station. Different measures of FE were subsequently calculated. For example, residual feed intake (RFI) was assumed to represent the residuals from a multiple regression model regressing FI on $\mathrm{ADG}$ and $\mathrm{BW}^{0.75}$ with batch included as a contemporary group (CG) effect. These data were described in full by Crowley et al. (2008). The second dataset consisted of information on CC, CF and CW on 2,566,969 animals slaughtered in 30 abattoirs in Ireland during the years 2005 to 2008. In the present study, the EUROP classification grades were transformed to a 15-point linear scale as outlined by Hickey et al. (2007), with a carcass fat score of 1 implying low fat coverage and a carcass conformation score of 1 implying poor conformation. Dam lactation number was grouped as $1,2,3$ to 4 and 5+. Animals with no sire information $(n=1,242,138)$ were discarded. Only males slaughtered between 300 and 1,200 days of age and females slaughtered between 300 and 875 days of age were retained. Only animals with Aberdeen Angus (AA), Belgian Blue (BB), Charolais (CH), Friesian (FR), Hereford (HE), Holstein (HO), Limousin (LM) and Simmental (SI) breed fractions were retained leaving 822,763 records. Additionally, animals where less than $75 \%$ of their breed fraction was known were discarded. Carcass weights less than $150 \mathrm{~kg}$ and greater than $550 \mathrm{~kg}(\mathrm{n}=11,150)$ were also omitted. Contemporary group was defined as herd by slaughter date and only CGs with 5 or more records were retained. A total of 386,729 individuals from 36,914 CGs remained. One quarter of CGs were randomly chosen to reduce the dataset size for variance component estimation. Genetic parameters were estimated using a linear mixed animal model in ASREML (Gilmour et al., 2007). Fixed effects included in the model for the carcass traits were CG, gender, non-linear association with age at slaughter, parity of dam, breed proportion, heterosis and recombination loss. An interaction between gender and age at slaughter was also fitted. Fixed effects included in the model for traits measured on the performance tested bulls were CG of test group, parity of dam, breed, age at the end and a quadratic effect of age at the end of test.

Results and Discussion Absolute genetic correlations between the performance test measures, excluding FE traits, and the three carcass traits ranged from 0.004 to 0.33 (Table 1). Although standard errors were large, ranging from 0.10 to 0.16 , they were considerably smaller than other studies with similar objectives (Nkrumah et al., 2007). Most of the genetic correlations with performance traits estimated in this study were not more than two standard errors from zero with the exception of the correlation between $\mathrm{CF}$ and $\mathrm{BW}$; selection for increased $\mathrm{BW}$ is expected to have a negative effect on $\mathrm{CF}$. With regard to the feed efficiency traits, $\mathrm{CC}$ and $\mathrm{CW}$ were both negatively correlated with FCR suggesting selection for improved (i.e., lower) FCR will improve both $\mathrm{CC}$ and $\mathrm{CW}$. Furthermore, CF and RFI were correlated indicating that selection for improved (i.e., lower) RFI will yield leaner carcasses. The lack of a genetic correlation between RFI and CW is somewhat expected given that RFI is phenotypically independent of BW.

Table 1 Genetic correlations of carcass traits with feed intake and weight measurements and measures of feed efficiency

\begin{tabular}{llllll}
\hline \hline & FI & BW & ADG $\boldsymbol{\Delta}$ & FCR $\boldsymbol{V}$ & RFI $\boldsymbol{~}$ \\
\cline { 2 - 6 } Carcass Conformation & $-0.12(0.12)$ & $-0.10(0.11)$ & $0.15(0.14)$ & $-0.33(0.14)$ & $-0.19(0.13)$ \\
Carcass Fat & $0.01(0.14)$ & $-0.33(0.11)$ & $-0.004(0.16)$ & $-0.02(0.17)$ & $0.32(0.14)$ \\
Carcass Weight & $0.01(0.11)$ & $0.08(0.10)$ & $0.19(0.13)$ & $-0.29(0.13)$ & $-0.17(0.12)$ \\
\hline \hline
\end{tabular}

$\boldsymbol{\Delta}=$ Indicates where a (more) positive value for this trait is desirable; $\boldsymbol{\nabla}=$ Indicates where a lesser value for this trait is desirable

Conclusion Selection for improved feed efficiency will not have any unfavourable repercussions for carcass traits. If anything selection for improved feed efficiency (i.e., lower FCR and lower RFI) will result in larger, leaner carcasses with better conformation. However the standard errors of the genetic correlations were large similar to previous studies, attributable mainly to the relatively small datasets for the FE variables. Therefore, there may be merit in pooling data from international sources to increase the dataset size and potentially generate more precise estimates of genetic parameters.

\section{References}

Crowley, J., Kenny, D.A., McGee, M., Crews Jr., D.H., Evans, R.D., Berry, D.P. 2008. Proc. Ag. Research Forum, Tullamore, 46.

Gilmour, A.R., Cullis, B.R., Welham, S.J., and Thompson, R. 2007. ASReml Reference Manual, $2^{\text {nd }}$ edition

Hickey, J.M., Keane, M.G., Kenny, D.A., Cromie, A.R. and Veerkamp, R.F. 2007. Journal of Animal Science 85, $314-321$.

Nkrumah, J.D., Basarab, J.A., Wang, Z., Li, C., Price, M.A., Okine, E.K., Crews Jr., D.H. and Moore, S.S. 2007. Journal of Animal Science 85, 2711-2720. 